\title{
The need for an evolutionary approach to ecotoxicology
}

To the Editor - It has been argued that that we are currently facing the sixth mass extinction event ${ }^{1}$, and the ubiquitous use of chemicals is one major driver for this loss of biodiversity ${ }^{2}$. Ecotoxicology, the estimation of environmental risks imposed by chemicals, is therefore an essential backbone for adequate mitigation efforts by stakeholders. However, if evolutionary biology is not taken into account in ecotoxicology, we may be asking the wrong questions in order to protect natural biodiversity. Here, we propose focusing on fitness, the essential factor governing wild populations, for ecotoxicological risk assessment (ERA).

Current ERA schemes appear flawed and outdated ${ }^{3}$ because they emphasize the mandatory endpoint measurement survival, despite evidence of sublethal effects being far more common and likely having severe consequences ${ }^{4}$. Focusing on longevity only becomes relevant if it ultimately enhances fitness. Irrespective of what the stressor may be, it therefore seems apparent that any reduction of fitness, and not survival alone, will have fundamental consequences for all species. Furthermore, false negative results, such as ecotoxicological studies yielding negative evidence for survival or even indicating enhanced survival ${ }^{5}$, but disguising a reduced fitness ${ }^{6}$, are likely to occur. Indeed, there are ample data showing clear negative effects of chemicals on fitness, but not on survival ${ }^{7}$, probably due to a trade-off between survival and reproduction ${ }^{8}$. It is therefore obvious that such a trade-off is paramount for any meaningful ERA. This also holds true for any measurement of sublethal effects, given they are no more relevant than mortality for the evaluation of fitness. Ultimately, any assessment of a sublethal parameter is only valid if it adequately reflects fitness. Therefore, conventional ecotoxicology tests are probably misleading for risk assessment schemes and will lead to inadequate policymaking. A change in mind set must occur in our community, because nature conservation requires an evolutionary approach.

Since field experiments are only used for 'higher tier' evaluations, as they are extremely difficult to control and standardize, future ERAs would need to be based only on measures of fitness in the laboratory. Fitness can be notoriously difficult to assess per se, especially under controlled laboratory conditions, where the well-being of an animal is often not met. Despite it now being common to have guidelines addressing the well-being of animals across all vertebrate $\operatorname{taxa}^{9}$, such practical implementations do not currently exist for invertebrates, in particular those providing essential ecosystem services. Given the major global declines in wild pollinating insects, this constitutes a major knowledge gap. Nevertheless, fitness can be practically incorporated in future ERAs and must be the gold standard, regardless of how challenging it may be. Laboratory procedures to estimate fitness indeed already exist for many species (for example, solitary bees ${ }^{6}$ ), which seem appropriate given the fitness of the laboratory-held controls matches respective field estimates. This value should be maintained over multiple generations to ensure a chemical has no significant long-term transgenerational effect. In some cases, laboratory assays may not allow the estimation of fitness, for example, for long-lived social insect colonies such as honeybees, for which fitness is the number of surviving swarms produced and the number of successfully mating males ${ }^{10}$. Honeybees are therefore not a suitable model species for Darwinian ecotoxicology tests. Instead, the model species for assessments must be selected based on their suitability for measuring fitness under laboratory conditions, in combination with established fitness data from the field. These species have to be chosen by stakeholders. One example for the practical implementation of Darwinian ERA is solitary bees, Osmia spp., for which fitness can be measured under laboratory conditions ${ }^{6}$, as females are expected to produce only $\sim 30$ offspring under favourable conditions ${ }^{11}$. Likewise, the fitness of eusocial bumblebees, Bombus spp., can easily be estimated by taking advantage of routine multi-generation rearing in the laboratory ${ }^{12,13}$. These two examples illustrate solid and feasible ways to measure fitness in the laboratory that transcend species boundaries at least for bees, thereby constituting two ideal models of future ERA. The identification of more suitable model species will clearly require substantial research, especially on the well-being of invertebrates, yet we must not shy away from meeting these challenges.

In conclusion, a shift to Darwinian ERA appears long overdue if our aim is to effectively mitigate the role of chemicals in the ongoing mass extinction of species. Policy decisions based on mortality estimates and sublethal effects other than fitness are probably leading to insufficient mitigation efforts. In light of limited resources, we therefore urge future ERA to focus exclusively on measures of fitness by using multi-generation reproductive studies in the laboratory. This would be a major step forward in ERA and enable reliable estimations of the environmental risks imposed by chemicals.

\section{Lars Straub, Verena Strobl and Peter Neumann (iD $凶$ \\ Institute of Bee Health, Vetsuisse Faculty, University of Bern, Bern, Switzerland.

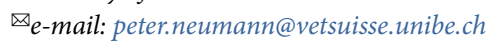

Published online: 23 April 2020 https://doi.org/10.1038/s41559-020-1194-6

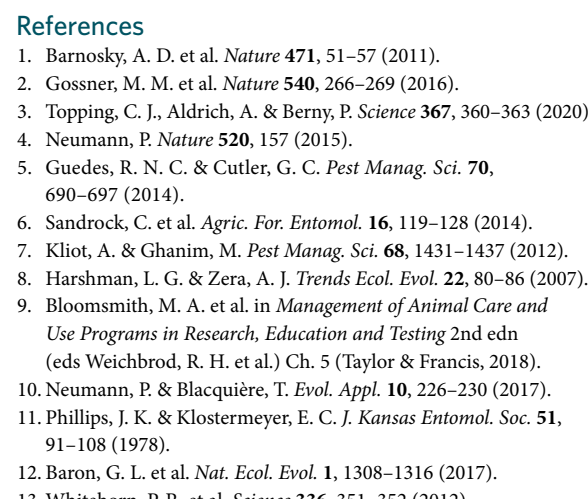

13. Whitehorn, P. R. et al. Science 336, 351-352 (2012).

Competing interests

The authors declare no competing interests. 